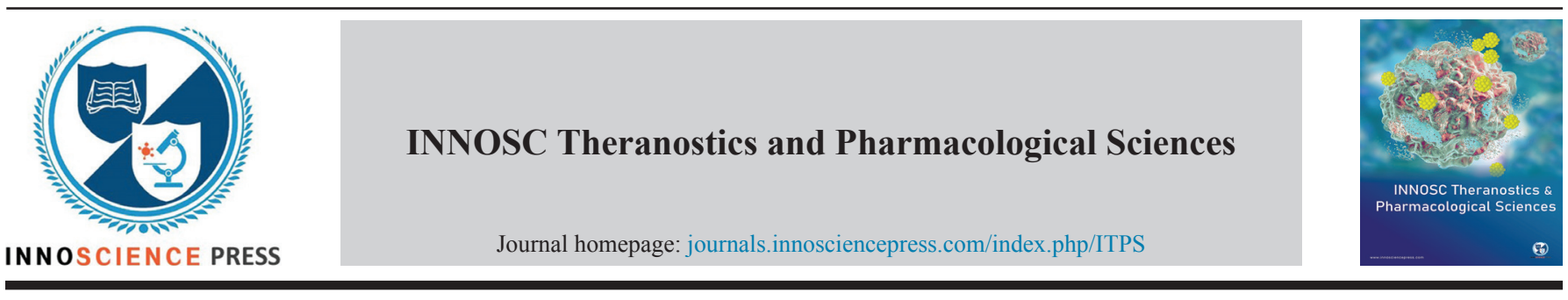

ORIGINAL ARTICLE

\title{
Skin Ultrasound as a Diagnostic Approach for Non-melanocytic Benign Skin Tumors
}

\author{
María Elena Del Prado ${ }^{*}$, Ricardo Ruiz-Villaverde ${ }^{2}$, Priscila Giavedoni $^{3}$, Ana Rodríguez $^{4}$, \\ David Vidal $^{5}$, Gastón Roustán ${ }^{6}$, Francisco Queipo ${ }^{7}$, Jose Aneiros-Fernandez ${ }^{8}$, \\ Paola del Castillo ${ }^{9}$, Elena Ruiz ${ }^{10}$, Irene Moysset ${ }^{5}$, Lola Suarez ${ }^{11}$, Laura Najera ${ }^{11}$, Fernando Alfajeme ${ }^{6}$ \\ ${ }^{1}$ Department of Dermatology, Hospital General San Jorge, Huesca, Spain \\ ${ }^{2}$ Department of Dermatology, Hospital Universitario San Cecilio, Granada, Spain \\ ${ }^{3}$ Department of Dermatology, Hospital Clínic, Barcelona, Spain \\ ${ }^{4}$ Department of Dermatology, Hospital La Paz, Madrid, Spain \\ ${ }^{5}$ Hospital de Sant Joan Despí Moisès Broggi, Barcelona, Spain \\ ${ }^{6}$ Department of Dermatology, Hospital Puerta de Hierro, Madrid, Spain \\ ${ }^{7}$ Hospital General San Jorge, Huesca, Spain \\ ${ }^{8}$ Department of Pathology, Hospital Universitario San Cecilio, Granada, Spain \\ ${ }^{9}$ Hospital Clínic, Barcelona, Spain \\ ${ }^{10}$ Hospital La Paz, Madrid, Spain \\ ${ }^{11}$ Hospital Puerta de Hierro, Madrid, Spain \\ *Corresponding Author: María Elena Del Prado, Email: melenadelprado@gmail.com
}

Received: September 11, 2020; Accepted: December 18, 2020; Published: December 30, 2020 DOI: 10.36922/itps.v3i2.944

Copyright: (c) 2020 Del Prado et al. This is an open-access article distributed under the terms of the Attribution NonCommercial 4.0 International 4.0 (CC BY-NC 4.0), which permits all non-commercial use, distribution and reproduction in any medium, provided the original work is properly cited.

\begin{abstract}
:
Background. The diagnostic imaging techniques in dermatology, such as skin ultrasound, could improve the diagnosis of nonmelanocytic benign tumor lesions, especially those of intradermal and subcutaneous nature.

Objective. The general objective of this study was to evaluate the validity and diagnostic safety of high-frequency ultrasound as a diagnostic test for non-melanocytic benign skin tumor detection. The specific objectives were to measure the sensitivity and specificity of ultrasound as a measure of the validity relative to the gold-standard test - diagnostic-therapeutic biopsy.

Methods. This study was carried out between January 2017 and June 2019. The patients ( $n=641)$ included in this study comprised of 318 women and 323 men who were between 48 and 74 years. All of them underwent an ultrasound examination of the skin tumor in the dermatology clinic, the removal of the non-melanocytic tumor, and a final histopathological examination.

Results. The skin ultrasound has a sensitivity and specificity of $93 \%$ and $88 \%$, respectively. Furthermore, the positive and negative predictive values of using skin ultrasound are $84 \%$ and $94 \%$, respectively.

Conclusion. Skin ultrasound is a diagnostic technique with high sensitivity and specificity in the diagnosis of non-melanocytic skin tumors.
\end{abstract}

Keywords: Skin ultrasound, Non-melanocytic benign skin tumor, Diagnosis 


\section{Introduction}

Before conducting this multicenter study, we conducted a retrospective study in which the pathological anatomy of the excised skin tumors was analyzed, and the initial diagnosis of which suggested that surgery was warranted. Of the 131 samples analyzed using the gold-standard test of pathological anatomy, $76(58 \%)$ of them were benign and surgery was not have required, whereas $55(42 \%)$ were malignant and the surgical treatment was indicated.

Considering these data, it is necessary to analyze the possibilities of high-resolution ultrasound of the skin in order to avoid unnecessary surgeries, discomfort to patients, and wasteful consumption of health system resources as a result of not having an accurate diagnostic technique. Since many of the excised lesions do not pose any risk of malignant transformation, a simple, bi-annual follow-up, or ultrasound examination to closely monitor their size would be sufficient, thus avoiding risks of infection of the surgical wound and primary nursing care as well as reducing the number of surgeries so as to minimize the consumption of public health resources.

Based on our bibliographic search in PubMed using "skin ultrasound" and "skin tumors" the search terms, we realized that the presence of increasingly better characterized ultrasound patterns [1] can, to a great extent, guide us in the clinical diagnosis of the tumors. The ultrasound patterns tend to associate with three principal types of skin tumors - epidermoid cyst, pilomatrixoma, and lipomas. An epidermoid cyst is a hypoechoic oval mass with a conduit that connects it to the surface called the punctum and with posterior acoustic reinforcement [2]. A pilomatrixoma is a hyperechoic solid mass with hypoechoic ring, showing punctate calcifications with posterior acoustic shadow. In the periphery, they may present some degree of vascularization. Lipomas are typical when their content is exclusively fatty and appear as markedly hyperechoic oval masses that follow the transverse axis of the skin layers. On the other hand, atypical lipomas are, in addition to their fat content, associated with connective tissue (fibrolipomas) or capillaries (angiolipomas) [3].
Ultrasound is originally a radiodiagnostic technique, but has progressively been used by other specialties. In dermatology, high-frequency probes $(>15 \mathrm{MHz})$ are the sought-after equipment for discriminating the echoes of skin components with resolution. The exploration probes must be linear, since we are interested in structures parallel to the body surface. Through the Doppler Effect which is the change in the frequency of the sound emitted when reflected on moving erythrocytes, we can detect blood flow in skin structures, which is increased in inflammation and tumors. Dermoscopy allows the early diagnosis of malignant melanoma and the description of patterns in nevi [4-6]. However, dermoscopy could not clearly detect benign cutaneous and subcutaneous tumors because they are covered by normal skin or do not present characteristic dermoscopic findings.

The introduction of new diagnostic techniques, such as skin ultrasound, has improved the sensitivity and specificity of the diagnosis of the benign tumor lesions, mainly subcutaneous, increasing their precision [7] up to almost $20 \%$. These techniques help us to better characterize the tumors (location and size) [8], their relationships with adjacent structures, and degree of vascularization, as well as to plan for surgeries indicated for those who require such as $[9,10]$. It allows differential diagnosis in clinically doubtful cases and assists minor outpatient surgery such as ultrasound-guided biopsies, drains, infiltrations, and removal of foreign bodies.

The general objective of this study was to evaluate the validity and diagnostic safety of highfrequency ultrasound as a diagnostic test for benign non-melanocytic benign skin tumor detection. The specific objectives were to measure the sensitivity and specificity of ultrasound as a measure of the validity relative to the gold-standard test diagnostic-therapeutic biopsy.

\section{Methods}

\subsection{Study design}

This is a prospective validation study where a cohort of subjects with undiagnosed nonmelanocytic tumors was selected for the skin ultrasound examination. Then, the tumors were removed and the gold-standard, histopathological test was performed according to usual practice. The 
prospective nature of the study and the fact that the test was applied before making the final diagnosis control the main biases, ensuring the independent evaluation of the test to be validated and the diagnostic test with which it is compared. All the subjects, regardless of their results, underwent the full diagnostic process. The results allowed us to measure the sensitivity and specificity as well as the positive and negative predictive values. The categories of the results would be dichotomous or trichotomous and the diagnoses may include many entities but ultimately they would be reduced to benign or malignant. Our intention was to apply all known ultrasound variables in the descriptions of skin tumors and to verify which have been the most significant for the diagnosis. In this study, the researchers were blind to the samples, they received for examination and clinical or relevant data that could possibly give rise to bias in their diagnosis were not revealed to them.

The current study was carried out according to the Good Clinical Practice that regulates the conduct of clinical studies, and the ethical principles established in the Declaration of Helsinki. All documents related to the study (including study protocols) were approved by an internal review board or independent research ethics committee of each of the participating hospitals and all patients gave their written informed consent before participating in the study.

\subsection{Study participants}

The patients were recruited and selected from the Dermatology Departments of six hospitals located in different Spanish cities. These hospitals are Hospital San Jorge de Huesca, Hospital Puerta de Hierro de Majadahonda in Madrid, Hospital de la Paz in Madrid, Hospital Vall de Hebron in Barcelona, Hospital de Sant Joan Despí Moissès Broggi in Barcelona, and Hospital Virgen de las Nieves in Granada. The recruitment and selection period was from February 1, 2018, to January 1, 2019.

The study participants were recruited on a pre-condition that the size of the non-melanocytic tumors must be in a range from $0.5 \mathrm{~mm}$ to $10 \mathrm{~cm}$, as determined by the lateral resolution of the Biosound MyLab 25 Portable Ultrasound (Universal Diagnostic Solutions, CA, USA). The study only considered and selected the participants based on the following selection criteria: (i) Patients older than 18 years with cutaneous and/ or subcutaneous non-melanocytic tumors which are amenable to surgery after clinical diagnosis according to the routine clinical practice; (ii) patients who had given the informed consent to perform complementary, non-invasive diagnostic imaging test (ultrasound), and surgical removal of the tumor for histopathological examination as per the consultation. The present study did not consider and select patients older than 18 years who presented with non-tumor inflammatory diseases or melanocytic tumors, such as nevus, and those who did not sign the informed consent forms allowing the procedures of skin ultrasound and/ or surgical removal of the tumor, despite fulfilling the selection criteria. The informed consent of the study participants was obtained. The study was approved by the Aragón Clinical Research Ethics Committee (CEICA; reference number: PI16/00726).

Ultrasound tests were performed on 641 patients from February 15, 2018, to February 7, 2019. Surgical removals of the tumors in this study were performed between February 15, 2018 and February 21, 2019, whereas the histopathological examination of the samples was carried out starting March 7, 2018.

\subsection{Skin ultrasound}

The current study used the ultrasound scan coupled with a $16-22 \mathrm{MHz}$ probe, which is considered using high frequency in dermatology. The ultrasound model used by all hospitals was Esaote My Lab 25 gold coupled with $18 \mathrm{MHz}$ probes. Ultrasound is a noninvasive technique that does not involve surgeries and obviate the need for anesthetics or radiotracers.

\subsection{Pathological examination}

The pathological study of skin tumors is based on the morphological assessment of the sections stained in hematoxylin and eosin. The services had complementary histochemical, immunohistochemical, or molecular pathology techniques available for doubtful cases in which the morphological variables selected by the pathologists were not sufficient. 


\subsection{Variables}

\subsubsection{Ultrasound variables}

Eight ultrasound variables were determined and selected based on the ultrasound descriptions in the literature about skin tumors, and the parameters of each variable were assigned as either benign (B) or malignant (M) (Panel 1). This is to discern the variables that could help us in the diagnosis of benignity or malignancy.

\subsubsection{Histopathological variables}

The group of pathologists specialized in dermatological six histopathological variables was determined and the parameters of each variable were assigned as either benign (B) or malignant (M) (Panel 2).

Panel 1. Ultrasound variables and their parameters

(B) indicate benignity and (M) malignancy

1. Margins

- Well defined (B)

- Badly defined (M)

2. Shape

- Round, oval, or regular (B)

- Lobulate, multilobed, or irregular (M)

3. Localization in tumor

- Superficial (epidermis and/or superficial dermis) (B)

- Deep (middle and deep dermis and/or subcutaneous tissue and/or fascia) (M)

4. Size (measured in $\mathrm{mm}$ in transversal and longitudinal planes)

- $<5 \mathrm{~mm}(\mathrm{~B})$

- $\geq \mathrm{B}$ mm (M)

5. Tumor echogenicity

- Homogeneous (a single echogenicity) (B)

- Heterogeneous (a combination of different echogenicities such as hypo-, hyper-, and isoechoic tumors) (M)

6. Echogenicity of peritumoral tissue

- Normal (B)

- Altered (M)

7. Vascularization of the tumor

- Absence or presence of unifocal peripheral vessels (B)

- Presence of multifocal intralesional or peripheral vessels (M)

8. Intralesional calcifications (well-defined intralesional or perilesional hyperechoic areas causing posterior acoustic shadowing)

- Present (B)

- Absent (M)

\subsection{Statistic analysis}

The categorical variables are expressed as counts and percentages. The data distribution of the continuous variables was tested using ShapiroWilks test to assess its normality. The continuous variables, if normally distributed, are expressed as mean and standard deviation. The continuous variables that do not follow a normal distribution are expressed as median, first quartile, and third quartile. The association between the factors and the histopathological test results or diagnosis was analyzed using Chi-squared or Fisher's exact tests for the categorical variables and Mann-Whitney $U$ test for the continuous variables.

Further analysis was carried out using univariate and multivariate logistic regression models. The odds ratio and $95 \%$ confidence interval of each variable are indicated. Sensitivity, specificity, predictive values, and likelihood ratios of the diagnostic tests were calculated.

The $\mathrm{R}$ software [11] and compare groups library [12] were used in the analysis.

\section{Results}

The baseline characteristics of the study population are shown in Table 1.

The univariate analysis of ultrasound variables showed that tumor size, tumor echogenicity of

Panel 2. Histopathological variables and their parameters

(B) indicate benignity and (M) malignancy

1. Growth

- Expansive (B)

- Infiltrative (M)

2. Necrosis

- Absent or present and scarce (B)

- Present and abundant (M)

3. Ulceration

- Absent or present and scarce (B)

- Present and abundant (M)

4. Nucleus/cytoplasm ratio

- Not augmented (B)

- Augmented (M)

5. Mitosis

- Rare typical or a rare atypical (B)

- Frequent typical or frequent atypical (M)

6. Distribution of vascularization

- Regular (B)

- Irregular (M) 
Table 1. Baseline characteristics of the study population

\begin{tabular}{|c|c|}
\hline & $n=641$ \\
\hline \multicolumn{2}{|l|}{ Study participants by hospitals } \\
\hline University Clinic Hospital & $162(25.27 \%)$ \\
\hline Sant Joan Despí Moisès Broggi Hospital & $20(3.12 \%)$ \\
\hline General Hospital San Jorge & $105(16.38 \%)$ \\
\hline La Paz University Hospital & $59(9.20 \%)$ \\
\hline $\begin{array}{l}\text { Puerta de Hierro Majadahonda } \\
\text { University Hospital }\end{array}$ & $144(22.46 \%)$ \\
\hline Virgen de las Nieves University Hospital & $151(23.56 \%)$ \\
\hline Age & $\begin{array}{c}63.00 \\
(48.00 ; 74.00)\end{array}$ \\
\hline \multicolumn{2}{|l|}{ Sex } \\
\hline Female & $318(49.61 \%)$ \\
\hline Male & $323(50.39 \%)$ \\
\hline \multicolumn{2}{|l|}{ Ultrasound variables } \\
\hline \multicolumn{2}{|l|}{ Margins } \\
\hline Well-defined & $461(71.92 \%)$ \\
\hline Badly defined & $180(28.08 \%)$ \\
\hline \multicolumn{2}{|l|}{ Shape } \\
\hline Oval & $348(54.29 \%)$ \\
\hline Rounded & $293(45.71 \%)$ \\
\hline Irregular & $247(38.53 \%)$ \\
\hline Regular & $394(61.47 \%)$ \\
\hline Lobulated & $589(91.89 \%)$ \\
\hline Multilobed & $52(8.11 \%)$ \\
\hline \multicolumn{2}{|l|}{ Location of tumor } \\
\hline Superficial & $369(57.57 \%)$ \\
\hline Deep & $272(42.43 \%)$ \\
\hline Size & $\begin{array}{c}8.00 \\
(6.00 ; 13.00)\end{array}$ \\
\hline$<5 \mathrm{~mm}$ & $70(10.92 \%)$ \\
\hline$\geq 1 \mathrm{~mm}$ & $571(89.08 \%)$ \\
\hline \multicolumn{2}{|l|}{ Tumor echogenicity } \\
\hline Homogeneous & $483(75.35 \%)$ \\
\hline Heterogeneous & $158(24.65 \%)$ \\
\hline \multicolumn{2}{|l|}{ Echogenicity of the peritumoral tissue } \\
\hline Normal & $486(75.82 \%)$ \\
\hline Altered & $155(24.18 \%)$ \\
\hline \multicolumn{2}{|l|}{ Vascularization of the tumor } \\
\hline Absent & $257(40.09 \%)$ \\
\hline Multifocal intralesional vessels & $152(23.71 \%)$ \\
\hline Multifocal peripheral vessels & $112(17.47 \%)$ \\
\hline Unifocal peripheral vessels & $120(18.72 \%)$ \\
\hline \multicolumn{2}{|l|}{ Intralesional calcifications } \\
\hline Absent & $592(92.36 \%)$ \\
\hline Present & $49(7.64 \%)$ \\
\hline
\end{tabular}

Table 1. (Continued)

$n=641$

Diagnosis based on the ultrasound variables

High suspicion of benignity

$345(53.82 \%)$

High suspicion of malignancy

$296(46.18 \%)$

Histopathological variables

Growth

Expansive

$427(66.61 \%)$

Infiltrative

$214(33.39 \%)$

Necrosis

Absent

$557(86.90 \%)$

Present and abundant

$15(2.34 \%)$

Present and scarce

$69(10.76 \%)$

Ulceration

Absent

$531(82.84 \%)$

Present and abundant

$40(6.24 \%)$

Present and scarce

$70(10.92 \%)$

Nucleus/cytoplasm ratio

Augmented

$280(43.68 \%)$

Not augmented

$361(56.32 \%)$

Mitosis

Rare atypical

$144(22.46 \%)$

Rare typical

$384(59.91 \%)$

Frequent atypical

$58(9.05 \%)$

Frequent typical

$55(8.58 \%)$

Distribution of vascularization

$\begin{array}{ll}\text { Irregular } & 278(43.37 \%) \\ \text { Regular } & 363(56.63 \%)\end{array}$

Diagnosis based on the histopathological variables

High suspicion of benignity

$371(57.88 \%)$

High suspicion of malignancy

$270(42.12 \%)$

the peritumoral tissue, and tumor vascularization were significantly associated with the diagnosis of tumor benignity and malignancy (Table 2). A tumor size $\geq 5 \mathrm{~mm}$ is 0.94 time more likely to predict malignancy than tumors smaller than 5 $\mathrm{mm}$. The results also demonstrate that tumors with altered echogenicity of the peritumoral tissue are 5.6 times more likely to predict malignancy than tumors with normal echogenicity of peritumoral tissue. With an odds ratio of 4.30, it was shown that tumors that are vascularized with multiple vessels inside and/or on the periphery of the tumor are associated with malignancy than tumors without vascularization or with only one vessel on the periphery of the tumor. Tumors 
Table 2. Univariate analysis of the ultrasound variables

\begin{tabular}{|c|c|c|c|c|c|}
\hline & $\begin{array}{c}\text { All } \\
\text { participants } \\
(n=641)\end{array}$ & $\begin{array}{c}\text { High suspicion } \\
\text { of Benignity } \\
(n=371)\end{array}$ & $\begin{array}{l}\text { High suspicion } \\
\text { of Malignancy } \\
\quad(n=270)\end{array}$ & OR $(95 \% \mathrm{CI})$ & $p$ \\
\hline Age & $\begin{array}{l}63.0(48.0- \\
74.0)\end{array}$ & $\begin{array}{l}52.0(40.0- \\
65.0)\end{array}$ & $72.5(65.0-81.0)$ & $1.09(1.08-1.11)$ & $<0.001$ \\
\hline \multicolumn{6}{|l|}{ Sex } \\
\hline Female & $318(49.6 \%)$ & $205(55.3 \%)$ & $113(41.9 \%)$ & Ref. & Ref. \\
\hline Male & $323(50.4 \%)$ & $166(44.7 \%)$ & $157(58.1 \%)$ & $1.71(1.25-2.36)$ & 0.001 \\
\hline \multicolumn{6}{|l|}{ Location of tumor } \\
\hline Superficial & $369(57.6 \%)$ & $206(55.5 \%)$ & $163(60.4 \%)$ & Ref. & Ref. \\
\hline Deep & $272(42.4 \%)$ & $165(44.5 \%)$ & $107(39.6 \%)$ & $0.82(0.60-1.13)$ & 0.222 \\
\hline \multicolumn{6}{|l|}{ Size } \\
\hline$<5 \mathrm{~mm}$ & $70(10.9 \%)$ & $50(13.5 \%)$ & $20(7.41 \%)$ & Ref. & Ref. \\
\hline$\geq 5 \mathrm{~mm}$ & $571(89.1 \%)$ & $321(86.5 \%)$ & $250(92.6 \%)$ & $1.94(1.14-3.41)$ & 0.014 \\
\hline \multicolumn{6}{|l|}{ Tumor echogenicity } \\
\hline Homogeneous & $483(75.4 \%)$ & $307(82.7 \%)$ & $176(65.2 \%)$ & Ref. & Ref. \\
\hline Heterogeneous & $158(24.6 \%)$ & $64(17.3 \%)$ & $94(34.8 \%)$ & $2.56(1.77-3.71)$ & $<0.001$ \\
\hline \multicolumn{6}{|c|}{ Echogenicity of the peritumoral tissue } \\
\hline Normal & $486(75.8 \%)$ & $329(88.7 \%)$ & $157(58.1 \%)$ & Ref. & Ref. \\
\hline Altered & $155(24.2 \%)$ & $42(11.3 \%)$ & $113(41.9 \%)$ & $5.61(3.78-8.47)$ & 0.000 \\
\hline \multicolumn{6}{|l|}{ Vascularization of the tissue } \\
\hline $\begin{array}{l}\text { Absent or unifocal peripheral } \\
\text { vessels }\end{array}$ & $377(58.8 \%)$ & $272(73.3 \%)$ & $105(38.9 \%)$ & Ref. & Ref. \\
\hline $\begin{array}{l}\text { Multifocal intralesional or } \\
\text { peripheral vessels }\end{array}$ & $264(41.2 \%)$ & $99(26.7 \%)$ & $165(61.1 \%)$ & $4.30(3.08-6.04)$ & 0.000 \\
\hline \multicolumn{6}{|c|}{ Diagnosis based on the ultrasound variables } \\
\hline High suspicion of benignity & $345(53.8 \%)$ & $325(87.6 \%)$ & $20(7.41 \%)$ & Ref. & Ref. \\
\hline High suspicion of malignancy & $296(46.2 \%)$ & $46(12.4 \%)$ & $250(92.6 \%)$ & $86.6(51.0-154)$ & 0.000 \\
\hline
\end{tabular}

OR: Odds ratio; CI: Confidence interval; $p$, P-value

with a heterogeneous echogenicity are 2.56 times more likely predict malignancy than tumors with homogeneous echogenicity. Despite not being a significant variable, the location of the tumor might be indicative of malignancy with deep lesions being able to suggest malignancy relative to the superficial ones. Figures 1-3 show the ultrasound scan images of some tumors.

The data of the ultrasound variables were also subject to further multivariate logistic regression analysis (Table 3). In this analysis, the statistically significant ultrasound variables, such as tumor size, echogenicity of the peritumoral tissue, and vascularization of the tumor, identified in the univariate analysis were confirmed. The tumors $\geq 5 \mathrm{~mm}$ have a risk of high suspicion of malignancy 2 times higher than the tumors smaller than $5 \mathrm{~mm}$. The altered echogenicity of the peritumoral tissue stands a risk 2.25 times higher than its normal counterpart of high suspicion of malignancy. Compared to tumors with no or unifocal peripheral vascularization, the tumors with multifocal peripheral or multifocal intralesional vascularization have a 2.4-fold increased risk of high suspicion of malignancy. The echogenicity of the tumor was a statistically significant variable in the univariate analysis, but in the multivariate logistic regression analysis, it was not a statistically significant ultrasound variable. This variation may be caused by the presence of several echogenicities within a malignant tumor.

In the subsequent analysis, the participants were classified according to tumor growth pattern for regression analysis (Table 4). We found that three 

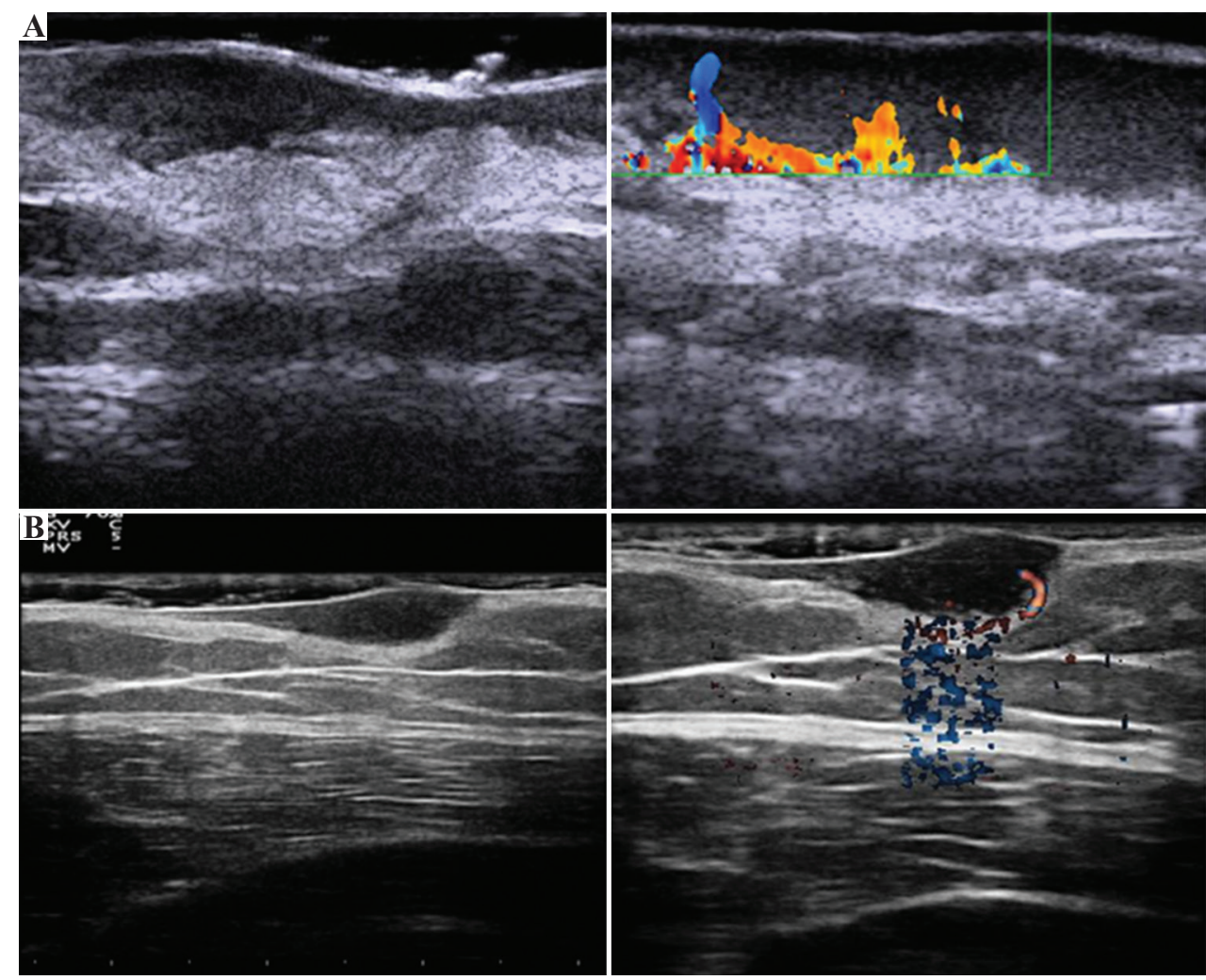

Figure 1. Ultrasound images of the dermatofibrosarcoma protuberans and dermohypodermic lesions. (A) Dermatofibrosarcoma protuberans and (B) dermohypodermic lesions. The images display impaired echogenicity of the surrounding tissue, a tumor size $>5 \mathrm{~mm}$, and abundant vascularization, pointing to high suspicion of malignancy.

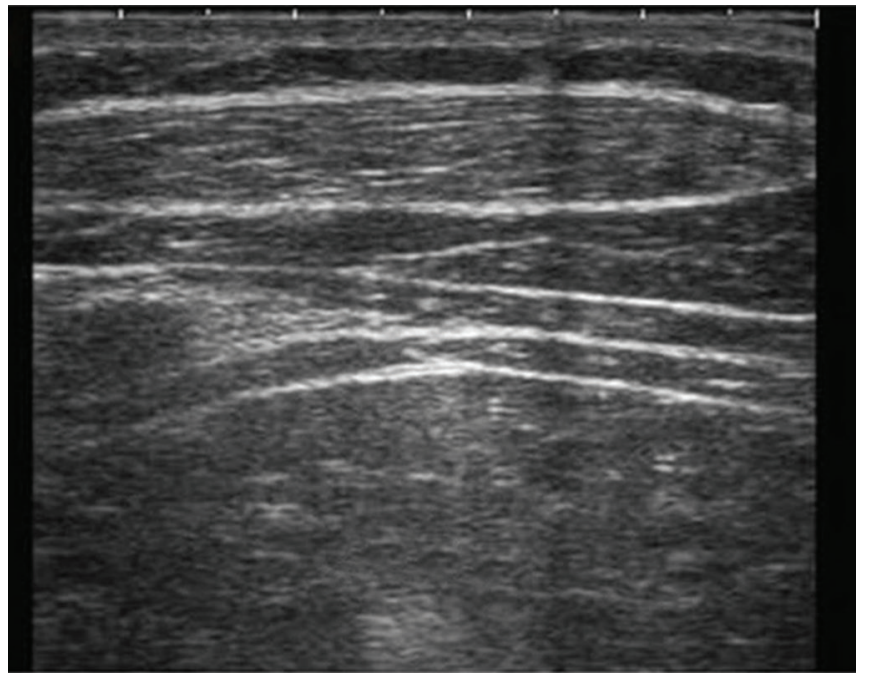

Figure 2. Ultrasound image of the subcutaneous lipoma. The image shows normal echogenicity of the peritumoral tissue and normal echogenicity within the isoechoic lesion but without vascularization, pointing to high suspicion of benignity.

ultrasound variables, such as tumor echogenicity, echogenicity of the peritumoral tissue, and vascularization of the tumor, were significantly

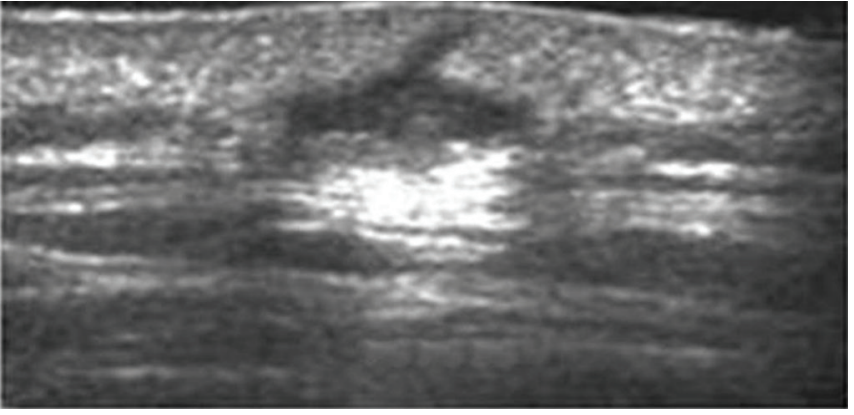

Figure 3. Ultrasound image of the epidermal cyst. The image displays a tumor size $<5 \mathrm{~mm}$ and normal echogenicity of the peritumoral tissue without vascularization and signs of infection or inflammation, indicating benignity of the cyst.

related to the tumor growth pattern. These data indicate that the tumor growth is correlated to the echogenicity of the peritumoral tissue.

The sensitivity and specificity of using skin ultrasound to detect non-melanocytic benign skin tumors are $93 \%$ and $88 \%$, respectively, and other indices pertaining its diagnostic performance can be found in Table 5. 
Table 3. Multivariate logistic regression analysis of the ultrasound variables

\begin{tabular}{|c|c|c|c|c|c|c|}
\hline & \multirow{2}{*}{$\begin{array}{c}\text { High suspicion } \\
\text { of benignity } \\
(n=371)\end{array}$} & \multirow{2}{*}{$\begin{array}{c}\text { High suspicion } \\
\text { of malignancy } \\
\quad(n=270)\end{array}$} & \multicolumn{2}{|c|}{ Univariate model } & \multicolumn{2}{|c|}{ Multivariate model } \\
\hline & & & OR $(95 \%$ CI $)$ & $p$ & OR $(95 \%$ CI $)$ & $p$ \\
\hline Age & $51.9(16.9 \%)$ & $71.8(11.9 \%)$ & $1.09(1.08-1.11)$ & $<0.001$ & $1.09(1.07-1.10)$ & $<0.001$ \\
\hline \multicolumn{7}{|l|}{ Sex } \\
\hline Woman & $205(55.3 \%)$ & $113(41.9 \%)$ & - & - & - & - \\
\hline Man & $166(44.7 \%)$ & $157(58.1 \%)$ & $1.72(1.25-2.36)$ & 0.001 & $1.73(1.15-2.60)$ & 0.008 \\
\hline \multicolumn{7}{|l|}{ Size } \\
\hline$<5 \mathrm{~mm}$ & $50(13.5 \%)$ & $20(7.4 \%)$ & - & - & - & - \\
\hline$\geq 5 \mathrm{~mm}$ & $321(86.5 \%)$ & $250(92.6 \%)$ & $1.95(1.15-3.42)$ & 0.016 & $2.09(1.08-4.17)$ & 0.031 \\
\hline \multicolumn{7}{|c|}{ Echogenicity of the peritumoral tissue } \\
\hline Normal & $329(88.7 \%)$ & $157(58.1 \%)$ & - & - & - & - \\
\hline Altered & $42(11.3 \%)$ & $113(41.9 \%)$ & $5.64(3.80-8.50)$ & $<0.001$ & $2.25(1.36-3.77)$ & 0.002 \\
\hline \multicolumn{7}{|l|}{ Tumor echogenicity } \\
\hline Homogeneous & $307(82.7 \%)$ & $176(65.2 \%)$ & - & - & - & - \\
\hline Heterogeneous & $64(17.3 \%)$ & $94(34.8 \%)$ & $2.56(1.78-3.71)$ & $<0.001$ & $1.07(0.63-1.81)$ & 0.787 \\
\hline \multicolumn{7}{|l|}{ Vascularization of tumor } \\
\hline $\begin{array}{l}\text { Absent or unifocal peripheral } \\
\text { vessels }\end{array}$ & $272(73.3 \%)$ & $105(38.9 \%)$ & - & - & - & - \\
\hline $\begin{array}{l}\text { Multifocal intralesional or } \\
\text { peripheral vessels }\end{array}$ & $99(26.7 \%)$ & $165(61.1 \%)$ & $4.32(3.09-6.06)$ & $<0.001$ & $2.44(1.56-3.82)$ & $<0.001$ \\
\hline
\end{tabular}

\section{Discussion}

The results of this prospective validation study on a cohort of subjects without a definitive diagnosis indicate that skin ultrasound is associated with a high sensitivity for the detection of non-melanocytic benign skin tumors, representing an important application in clinical practice. A high accuracy in tumor detection may help avoid unnecessary skin biopsies and surgeries or unfavorable scheduling for treatment, thereby reducing the waiting times for surgery and indirectly improving efficiency in the healthcare systems. The frequency of complications associated with surgeries, such as infections, wound dehiscence, and keloids is expected to reduce.

In the present study, the statistically significant ultrasound variables were the size of the tumor, echogenicity of the peritumoral tissue, and vascularization of the tumor. These data correspond to the ultrasound descriptions of malignant tumors described in the literature, such as those pertaining to squamous cell carcinoma, dermatofibrosarcoma protuberans, Merkel cell carcinoma [13], and basal cell carcinoma [14]. The ultrasound variables suggestive of benignity also correspond to the descriptions of benign tumors described in the literature [15].

In this study, we identified three ultrasound variables that are indicative of non-melanocytic benign skin tumors; particularly, a tumor size of $<5 \mathrm{~mm}$, normal echogenicity of the peritumoral tissue, and the absence or unifocal peripheral vascularization of the tumor are suggestive of benign skin tumors. On the other hand, a tumor size of $\geq 5 \mathrm{~mm}$, altered echogenicity of the peritumoral tissue, and multifocal peripheral or multifocal intralesional vascularization of the tumor are the ultrasound variables that are indicative of nonmelanocytic malignant skin tumors.

Despite not found to have statistically significant association with benignity and malignancy, certain ultrasound variables should be taken into account when making a diagnostic judgment of high suspicion of benignity or malignancy. Homogeneous and heterogeneous tumor echogenicities are in favor of the high suspicions of benignity and malignancy, respectively. The 
Table 4. Relationship of tumor growth with ultrasound variables

\begin{tabular}{|c|c|c|c|c|c|}
\hline & $\begin{array}{c}\text { All } \\
\text { participants } \\
(n=641)\end{array}$ & $\begin{array}{c}\text { Expansive } \\
\text { growth } \\
(n=427) \\
\end{array}$ & $\begin{array}{c}\text { Infiltrative } \\
\text { growth } \\
(n=214) \\
\end{array}$ & OR $(95 \% \mathrm{CI})$ & $p$ \\
\hline Age & $\begin{array}{c}63.0 \\
(48.0-74.0)\end{array}$ & $\begin{array}{c}60.0 \\
(46.0-71.0)\end{array}$ & $\begin{array}{c}68.0 \\
(50.0-76.0)\end{array}$ & $1.02(1.01-1.03)$ & 0.001 \\
\hline \multicolumn{6}{|l|}{ Sex } \\
\hline Female & $318(49.6 \%)$ & $210(49.2 \%)$ & $108(50.5 \%)$ & Ref. & Ref. \\
\hline Male & $323(50.4 \%)$ & $217(50.8 \%)$ & $106(49.5 \%)$ & $0.95(0.68-1.32)$ & 0.759 \\
\hline \multicolumn{6}{|l|}{ Location } \\
\hline Superficial & $369(57.6 \%)$ & $249(58.3 \%)$ & $120(56.1 \%)$ & Ref. & Ref. \\
\hline Deep & $272(42.4 \%)$ & $178(41.7 \%)$ & $94(43.9 \%)$ & $1.10(0.79-1.53)$ & 0.589 \\
\hline \multicolumn{6}{|l|}{ Size } \\
\hline$<5 \mathrm{~mm}$ & $70(10.9 \%)$ & $48(11.2 \%)$ & $22(10.3 \%)$ & Ref. & Ref. \\
\hline$\geq 5 \mathrm{~mm}$ & $571(89.1 \%)$ & $379(88.8 \%)$ & $192(89.7 \%)$ & $1.10(0.65-1.91)$ & 0.723 \\
\hline \multicolumn{6}{|l|}{ Tumor echogenicity } \\
\hline Homogeneous & $483(75.4 \%)$ & $340(79.6 \%)$ & $143(66.8 \%)$ & Ref. & Ref. \\
\hline Heterogeneous & $158(24.6 \%)$ & $87(20.4 \%)$ & $71(33.2 \%)$ & $1.94(1.34-2.81)$ & $<0.001$ \\
\hline \multicolumn{6}{|l|}{ Tumor echogenicity (hypoechoic tumor) } \\
\hline No & $73(11.4 \%)$ & $53(12.4 \%)$ & $20(9.35 \%)$ & Ref. & Ref. \\
\hline Yes & $568(88.6 \%)$ & $374(87.6 \%)$ & $194(90.7 \%)$ & $1.37(0.81-2.41)$ & 0.252 \\
\hline \multicolumn{6}{|l|}{ Tumor echogenicity (hyperechoic tumor) } \\
\hline No & $464(72.4 \%)$ & $320(74.9 \%)$ & $144(67.3 \%)$ & Ref. & Ref. \\
\hline Yes & $177(27.6 \%)$ & $107(25.1 \%)$ & $70(32.7 \%)$ & $1.45(1.01-2.08)$ & 0.043 \\
\hline \multicolumn{6}{|l|}{ Tumor echogenicity (isoechoic tumor) } \\
\hline Yes & $64(9.98 \%)$ & $36(8.43 \%)$ & $28(13.1 \%)$ & Ref. & Ref. \\
\hline No & $577(90.0 \%)$ & $391(91.6 \%)$ & $186(86.9 \%)$ & $0.61(0.36-1.04)$ & 0.070 \\
\hline \multicolumn{6}{|l|}{ Echogenicity of the peritumoral tissue } \\
\hline Normal & $486(75.8 \%)$ & $352(82.4 \%)$ & $134(62.6 \%)$ & Ref. & Ref. \\
\hline Altered & $155(24.2 \%)$ & $75(17.6 \%)$ & $80(37.4 \%)$ & $2.80(1.93-4.07)$ & $<0.001$ \\
\hline \multicolumn{6}{|l|}{ Vascularization of the tumor } \\
\hline $\begin{array}{l}\text { Absence or presence of unifocal } \\
\text { peripheral vessels }\end{array}$ & $377(58.8 \%)$ & $272(63.7 \%)$ & $105(49.1 \%)$ & Ref. & Ref. \\
\hline $\begin{array}{l}\text { Presence of multifocal intralesional or } \\
\text { peripheral vessels }\end{array}$ & $264(41.2 \%)$ & $155(36.3 \%)$ & $109(50.9 \%)$ & $1.82(1.30-2.54)$ & $<0.001$ \\
\hline \multicolumn{6}{|l|}{ Diagnosis based on the ultrasound variables } \\
\hline High suspicion of benignity & $345(53.8 \%)$ & $270(63.2 \%)$ & $75(35.0 \%)$ & Ref. & Ref. \\
\hline High suspicion of malignancy & $296(46.2 \%)$ & $157(36.8 \%)$ & $139(65.0 \%)$ & $3.18(2.26-4.50)$ & $<0.001$ \\
\hline
\end{tabular}

OR: Odds ratio; CI: Confidence interval; $p, \mathrm{P}$-value

reason why tumor echogenicity was not an ultrasound variable to be considered is because both classes of benign and malignant tumors can similarly possess the heterogeneous echogenicity. Typically, the malignant tumors, such as basal cell carcinomas, dermatofibrosarcoma protuberans, fibrosarcomas, and cutaneous lymphomas, are associated with heterogeneous echogenicity. Notably, however, the benign tumors, such as inclusion cysts, angiofibromas, myelolipomas, and evolving hematomas, also possess heterogeneous echogenicity, which might hinder the classification process. Similar to the tumor echogenicity variable, the tumor location variable might also not be able to provide an indecisive classification of the tumors, simply based on whether the tumor is on the epidermis or deep in the dermis or hypodermis. For instance, certain benign tumors such as lipomas and inclusion cysts can be found deep in the hypodermis or dermis, in contrast to the 
Table 5. Diagnostic utility of skin ultrasound for the detection of non-melanocytic benign skin tumors.

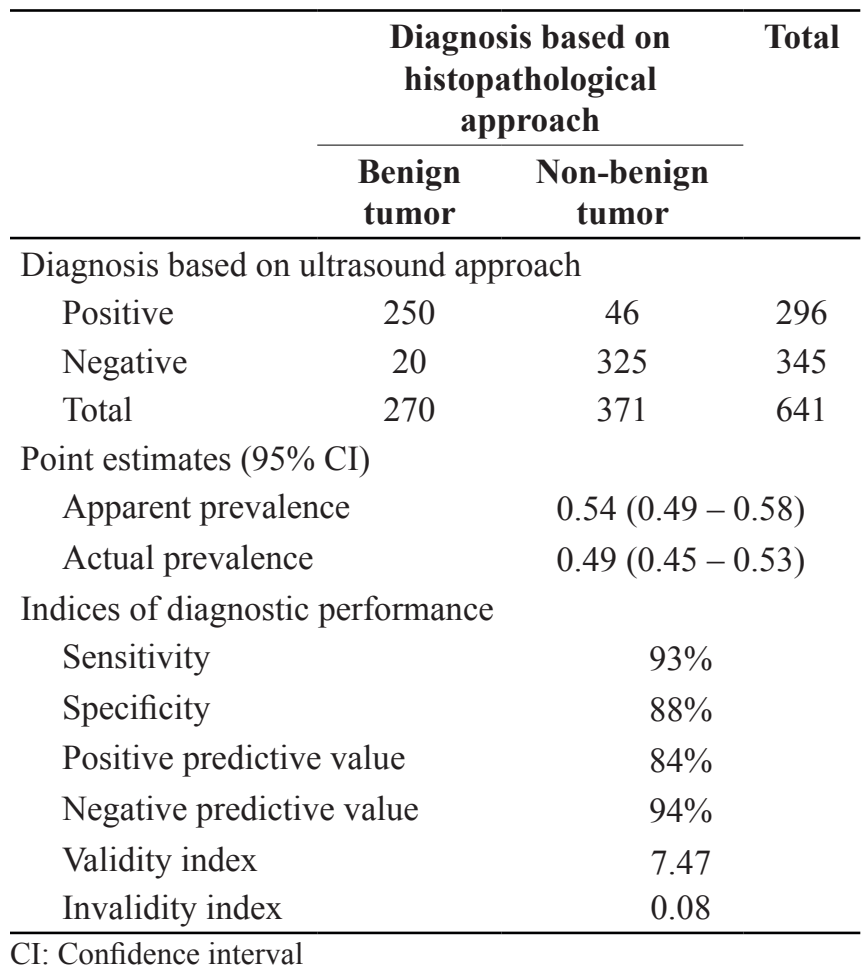

seborrheic keratoses and dermatofibromas that are located in the epidermis and superficial dermis. Furthermore, certain malignant tumors such as superficial spreading basal cell carcinoma and in situ squamous cell carcinomas are located in the epidermis and superficial dermis.

Furthermore, contrary to the established belief that benign tumors usually have well defined margins, benign tumors such as dermatofibroma have irregular borders, and ruptured cysts on ultrasound scan. Tumor shape is another ultrasound variable that does not helpfully contribute to the detection of benign skin tumor. Unlike the roundand oval-shaped benign tumors such as cysts and lipomas, lymphangiomas are actually lobulated or multilobed. Besides, malignant tumors which are theoretically to have multilobed shape (e.g., dermatofibrosarcoma protuberans) may have acquired round or oval shapes (e.g., nodular basal cell carcinomas).

Intralesional calcification occurs in both benign tumors such as inclusion cysts [3] and malignant tumors such as basal cell carcinomas [14]; therefore, this parameter is not specific to either type of the tumor. Specifically, the posterior acoustic shadow, which is a characteristic of hyperkeratotic or solid lesions that do not let sounds pass, occurs in both benign tumors (e.g., seborrheic keratosis, vulgar warts, and pilomatrixoma) [16] and malignant tumors (e.g., squamous cell carcinoma) [13]. The nonspecificity of the intralesional calcification also extends to posterior enhancement which is a characteristic in lesions with liquid content. In the same way, benign tumors such as inclusion cysts [3] and simple angiomas [16] and malignant tumors such as angiosarcomas are similarly associated with posterior enhancement.

The limitation of the present study was the potential information bias due to the fact that the same dermatologist performed the ultrasound examination and surgical removal of tumor, which should ideally be done by different professionals. This bias was resolved by randomizing because the test to be validated had a result independent of histopathology. In addition, masking techniques were used so that the dermatologists were aware of the description of the tumor samples they received.

\section{Conclusion}

With high diagnostic performance, skin ultrasound can be used as a complementary and noninvasive test for the detection of non-melanocytic benign skin tumors and the screening of suspected nonmelanocytic malignant tumors.

\section{Acknowledgments}

This study was funded by the PI16/project 00726 (Carlos III Health Institute) and the European Regional Development Fund: "A way to make Europe."

We thank four members from Instituto Aragonés de Ciencias de la Salud for their assistance in carrying out the present study: Anselmo Cabañas for the methodological support, Daniel Bordonaba for his assistance in statistical analysis, Mónica Torrijos for her assistance in matters pertaining the research ethics, and Pilar Lainez for assistance in data collection.

\section{Conflicts of interest}

The authors declare that they have no conflicts of interest. 


\section{Author contributions}

M.E.D.P, R.R-V., P.G., A.R., D.A., G.R., and F.A. are the dermatologists that gathered relevant data for the study. F.Q., J.A-F., P.C., E.R., I.M., L.S., and L.N are the anatomic pathologists that gathered relevant data for the study. M.E.D.P, R.R-V., and F.A. wrote the paper. M.E.D.P reviewed drafts of manuscript. All authors read and approved the final manuscript.

\section{References}

[1] Kuwano, Y.; Ishizaki, K.; Watanabe, R.; Nanko, H. Efficacy of Diagnostic Ultrasonography of Lipomas, Epidermalcysts, and Ganglions. Arch. Dermatol., 2009, 145, 761-4.

[2] Jin, W.; Ryu, K.N.; Kim, G.Y.; Kim, H.C.; Lee, J.H.; Park, J.S. Sonographic Findings of Ruptured Epidermal Inclusion Cysts in Superficial Soft Tissue: Emphasison Shapes, Pericystic Changes, and Pericystic Vascularity. J. Ultrasound Med., 2008, 27, 171-6.

[3] Gonzalez, C.P. Characterization of Dermatological Lesions by Ultrasound. Rev. Colomb. Radiol., 2014, 25(3), 4006-14.

[4] Annessi, G.; Bono, R.; Sampogna, F.; Faraggiana, T.; Abeni, D. Sensitivity, Specificity, and Diagnostic Accuracy of Three Dermoscopic Algorithmic Methods in the Diagnosis of Doubtful Melanocytic Lesions: The Importance of Light Brown Structureless Areas in Differentiating Atypical Melanocytic Nevi from thin Melanomas. J. Am. Acad. Dermatol., 2007, 56(5), 759-67.

[5] Pralong, P.; Bathelier, E.; Dalle, S.; Poulalhon, N.; Debarbieux, J. Dermoscopy of Lentigo Malign Melanoma: Report of 125 Cases. Br. J. Dermatol., 2012, 167(2), 280-7

[6] Fonseca, M.; Marchetti, M.A.; Chung, E.; Dusza, S.W.;
Burnett, M.E.; Marghoob, A.A.; Geller, A.C.; Bishop, M.; Scope, A.; Halpern, A.C. Cross-sectional Analysis of the Dermoscopic Patterns and Structures of Melanocytic Naevi on the Back and Legs of Adolescents. Br. J. Dermatol., 2015, 173(6), 1486-93.

[7] Alfageme, F. Practical Applications of Ultrasound Skin. Skin, 2012, 27, 204-9.

[8] Minds, O.; Oysul, A.; Harlak, A.; Zeybek, N.; Kozak, O.; Tufan, T. Ultrasonography Accurately Evaluates the Dimension and Shape of the Pilonidal Sinus. Clinics (Sao Paolo), 2009, 64, 189-92.

[9] Wortsman, X.; Worstman, J. Clinical Usefulness of Variable Frequency Ultrasound in Localized Lesions of the Skin. J. Am. Acad. Dermatol., 2010, 62, 247-56.

[10] Wortsman, X. Common Applications of Dermatologic Sonography. J. Ultrasound Med., 2012, 31, 97-111.

[11] R Core Team. A: A Language and Environment for Statistical Computing. R Foundation for Statistical Computing, Vienna, Austria, 2019.

[12] Subirana, I.; Sanz, H.; Vila, J. Building Bivariate Tables: The Compare Groups Package for R. J. Stat. Softw., 2014, 57(12), $1-16$.

[13] Hernández, C.; Aguilar, M.; de Troya, M. Ultrasound Applied to the Management of Non-melanoma Skin Cancer. Actas Dermosifiliogr., 2015, 106 Suppl 1, 21-8.

[14] Hernández-Ibáñez, C.; Blazquez-Sánchez, N.; Aguilar-Bernier, M.; Fúnez-Liébana, R.; Rivas-Ruiz, F.; de Troya-Martín, M. Usefulness of Cutaneous Ultrasound in the Classification of Subtypes of Primary Basal Cell Carcinomas. Usefulness of High-Frequency Ultrasound in the Classification of Histologic Subtypes of Primary Basal Cell Carcinoma. Actas Dermosifiliogr., 2017, 108(Suppl 1), $42-51$.

[15] Ruiz-Villaverde, R.; Arias-Santiago, S.; Repiso-Jiménez, J.B. Ultrasound in the Management of Benign Cutaneous Tumors. Actas Dermosifiliogr., 2015, 106(Suppl 1), 29-40.

[16] Whittle, C.; Baldassare, G. Ultrasonografia de Piel y Anexos. Rev. Chilena Radiol., 2004, 10(2), 81-88. 